\title{
A study of additional forms of the human sperm lactate dehydrogenase isoenzyme
}

\author{
M. Gavella and V. Lipovac \\ Vuk Vrhovac Institute of Diabetes, Endocrinology and Metabolic Diseases, Medical School, \\ University of Zagreb, Krijesnice bb, YU-41000 Zagreb, Yugoslavia
}

\begin{abstract}
Summary. Unusual isoenzymes of the sperm-specific lactate dehydrogenase detected in the seminal plasma of some infertile men were investigated using selective precipitation by antisera followed by electrophoretic resolution. The presence of two $\mathrm{C}$ subunit types was established: type $I$ is a polymer of the four common C-subunits and type II consists of four $\mathrm{C}^{*}$-subunits. Subunits of types I and II combine to form 5 sperm-specific binomially distributed lactate dehydrogenase isoenzymes. These results suggest the existence of two alleles at the c locus.
\end{abstract}

\section{Introduction}

Human isoenzyme LDH-X is a homotetramer composed of four C-subunits ( $\mathrm{LDH}-\mathrm{C}_{4}$ ) synthesized in primary spermatocytes to fulfil a very special function for the metabolic requirements of mature spermatozoa (Wheat \& Goldberg, 1983). According to the literature surveys reported by Markert

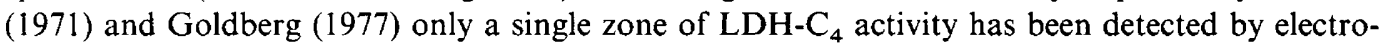
phoresis in spermatozoa, testes or seminal plasma. However, Gavella et al. (1984) detected an unusual LDH isoenzyme pattern in the testes, spermatozoa and seminal plasma of some infertile men, suggesting the presence of heterotetramers probably of the B-C type because of the great structural and conformational similarity between the C- and B-subunits (Goldberg, 1977) and their similar resistance to denaturation (Wilkinson, 1970).

The aim of this paper is to report our further work on the identification of the tetramer combination by using immunochemical and heat-inactivation methods.

\section{Materials and Methods}

Material. Semen samples of infertile men, in which the presence of new bands of the LDH-C $\mathrm{C}_{4}$ isoenzymes had been detected (this pattern had been found in 20 out of 1200 semen samples obtained from infertile men) and semen samples of healthy fertile subjects were studied. All of these 20 patients had been living in childless marriages for several years. Their sperm concentration was within the normal range $\left(41-83 \times 10^{6} / \mathrm{ml}\right)$ but sperm motility was considerably reduced $(13-34 \%)$. Healthy fertile subjects were men whose wives, at the time the study started, were already pregnant.

The semen samples were collected by masturbation after 4 days of sexual abstinence. The samples were allowed to liquefy at room temperature, then centrifuged at $800 \mathrm{~g}$ for $10 \mathrm{~min}$ and the supernatant, i.e. seminal plasma, was stored at $-20^{\circ} \mathrm{C}$ until analysed.

Reagents. Acrylamide, $N, N^{\prime}$-methylendiacrylamide, ammonium persulphate, sodium lactate and standard analytical grade laboratory reagents purchased from Merck (Darmstadt, FRG) and phenazine methosulphate, nitrobluetetrazolium salt and 2-oxohexanoate obtained from Sigma (St Louis, MO, U.S.A.) were used. Rabbit anti-LDH-C 4 serum was obtained from Professor E. Goldberg as a gift. For the elimination of isoenzymes containing A-subunits we used

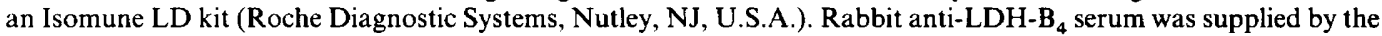
Institute of Immunology (Zagreb, Yugoslavia) where rabbits had been immunized with LDH-1 isoenzyme from bovine heart (type IX; Sigma).

Methods. Lactate dehydrogenase isoenzymes were detected by electrophoresis on $7 \%$ polyacrylamide gel by a modification of the method of Davis (Gavella et al., 1982), using a $3 \mathrm{~mA}$ current per tube for 60 min at room temperature. The gels were stained by incubation at $37^{\circ} \mathrm{C}$ in a medium containing lactate, NAD, phenazine methosulphate 


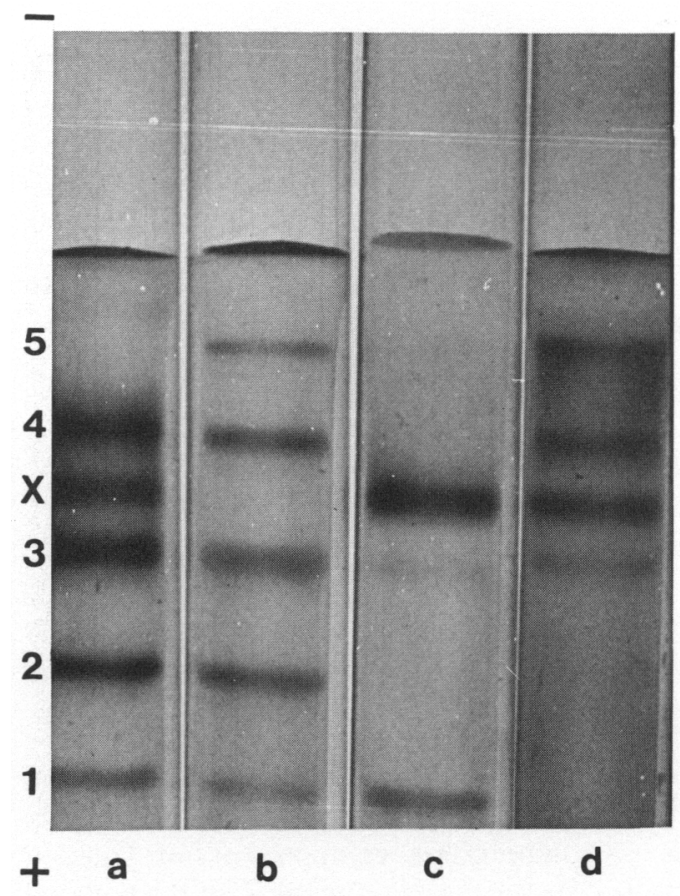

Fig. 1. Lactate dehydrogenase isoenzymes in normal human seminal plasma; (a) usual electrophoretic pattern; (b) seminal plasma incubated with antiserum to $\mathrm{LDH}^{-\mathrm{C}_{4}}$ before electrophoresis; (c) seminal plasma with antiserum to LDH- $\mathrm{A}_{4}$; (d) seminal plasma with antiserum to LDH-B . $^{2}$

and nitrobluetetrazolium salt, and then immersed in a fixing solution of $5 \%$ acetic acid. Relative percentages of LDH isoenzymes were quantified using a Chemetron densitometer.

Specific $\mathrm{LDH}-\mathrm{C}_{4}$ activity was determined using 2-oxohexanoate as substrate according to the method of Burgos et al. (1979).

$\mathrm{LDH}$ isoenzymes containing $\mathrm{C}$ - or $\mathrm{B}$-subunits were removed by rabbit anti- $\mathrm{LDH}-\mathrm{C}_{4}$ and anti- $\mathrm{LDH}-\mathrm{B}_{4}$ serum, respectively. LDH isoenzymes containing A-subunits were removed from the semen samples by the method of Usategui-Gomez et al. (1979), using the reagents from the Isomune LD kit. This method includes immunochemical precipitation of isoenzymes LDH-2 to LDH-5. In this assay, an antiserum raised in goats against purified LDH-5 from monkey muscle is used, and the antigen-antibody complexes formed are precipitated by adding donkey antigoat gamma globulin immobilized on inert polymer.

The samples were heat-inactivated by incubation at $56^{\circ} \mathrm{C}, 65^{\circ} \mathrm{C}$ and $70^{\circ} \mathrm{C}$ for various times and then cooled on ice and analysed by electrophoresis.

\section{Results}

Figure 1(a) shows the usual pattern of human seminal plasma in which 6 sites of LDH activity are demonstrable: isoenzymes LDH-1 to LDH-5, and $\mathrm{LDH}-\mathrm{C}_{4}$ which is located between LDH-3 and $\mathrm{LDH}-4$. By selective precipitation by antibodies, followed by electrophoretic resolution of the unprecipitated isoenzymes, the following patterns were obtained. (1) Rabbit anti-LDH-C 4 serum completely and specifically removed isoenzyme $\mathrm{C}_{4}$ from the seminal plasma (Fig. 1b). (2) AntiLDH- $\mathrm{A}_{4}$ serum removed all $\mathrm{LDH}$ isoenzymes containing A-subunits, while the homotetramers containing $\mathrm{B}_{4}$ (LDH-1) and $\mathrm{C}_{4}$-subunits remained unaltered (Fig. 1c). (3) Antiserum to LDH-B removed the activity of $\mathrm{LDH}$ isoenzymes containing $\mathrm{B}$ subunits (the $\mathrm{LDH}-1$ and $\mathrm{LDH} 2$ isoenzymes were completely removed, whereas $\mathrm{LDH}-3$ became markedly weaker, but did not affect the LDH-C band) (Fig. 1d). 


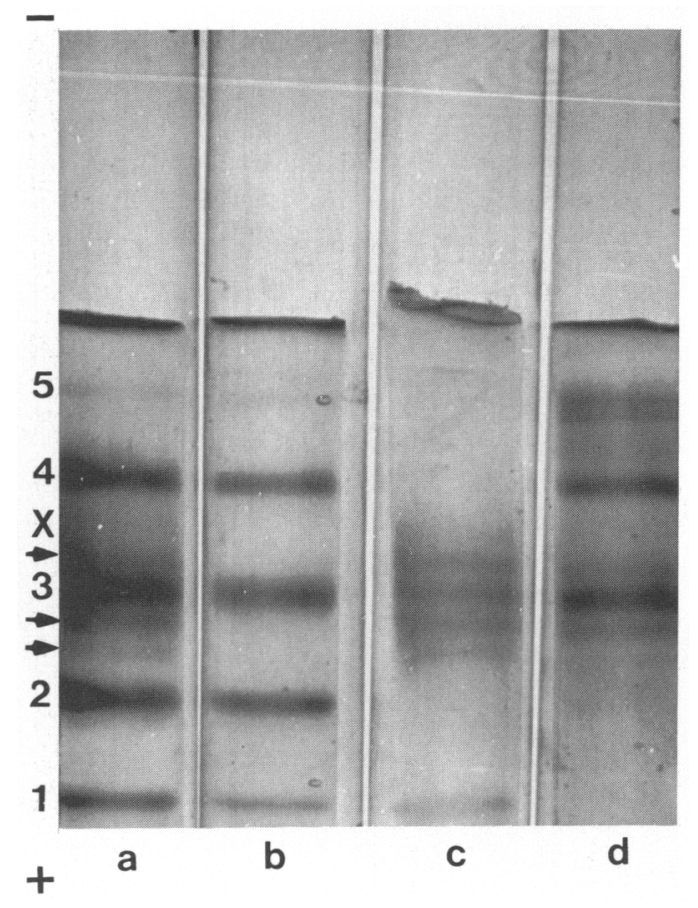

Fig. 2. Lactate dehydrogenase isoenzymes in seminal plasma from infertile men: (a) unusual electrophoretic pattern; the arrows indicate 3 additional bands; (b) seminal plasma with anti-

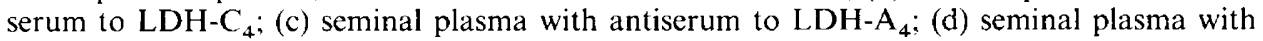
antiserum to $\mathrm{LDH}^{-\mathrm{B}_{4}}$.

Figure 2(a) illustrates a pattern of human seminal plasma with 9 sites of LDH activity. The three additional bands near LDH-3 $\left(\mathrm{A}_{2} \mathrm{~B}_{2}\right)$ are denoted with arrows. Two bands appeared between LDH-2 and LDH-3, and one migrated between $\mathrm{LDH}-3$ and $\mathrm{LDH}-\mathrm{C}_{4}$. In addition, the intensity of $\mathrm{LDH}-\mathrm{C}_{4}$ decreased. Using specific antibodies, the following patterns were obtained. (1) Antiserum to $\mathrm{LDH}_{-} \mathrm{C}_{4}$ removed these new bands along with $\mathrm{LDH}-\mathrm{C}_{4}$, while the LDH-1 to LDH-5 bands remained distinct (Fig. 2b). (2) Anti- $\mathrm{LDH}^{-\mathrm{B}_{4}}$ serum eliminated the activity of isoenzymes containing B-subunits, but the unusual pattern near LDH-3 remained unchanged (Fig. 2d). Incubation of seminal plasma with anti- $\mathrm{A}_{4}$ resulted in a selective removal of $\mathrm{LDH}$ isoenzymes containing A-subunits, so that LDH-1 $\left(\mathrm{B}_{4}\right)$, a weak fraction at the site of $\mathrm{LDH}-\mathrm{C}_{4}$ and the three additional electrophoretic bands remained. However, it was surprising that $A_{2} B_{2}$ isoenzyme was not removed (Fig. 2c), unlike in normal seminal plasma, where this fraction was eliminated or strongly reduced (Fig. Ic). At first we thought that $\mathrm{A}_{2} \mathrm{~B}_{2}$ perhaps required a higher concentration of anti- $\mathrm{A}_{4}$ serum for complete inhibition, but the pattern did not change with increased concentration of antiserum, suggesting the existence of a tetramer with the same electrophoretic mobility as LDH-3 and actually occupying the same position on the gel.

For further characterization of the additional isoenzymes detected in the semen, heat inactivation experiments were carried out. These experiments also showed differences in the behaviour of the additional bands near LDH-3 and at LDH-3 between normal and pathological ejaculates. It has been well documented that the cathode-migrating $\mathrm{LDH}$ isoenzymes containing A-subunits are less stable and can be more readily eliminated than the fractions containing B-subunits, which are more stable. 


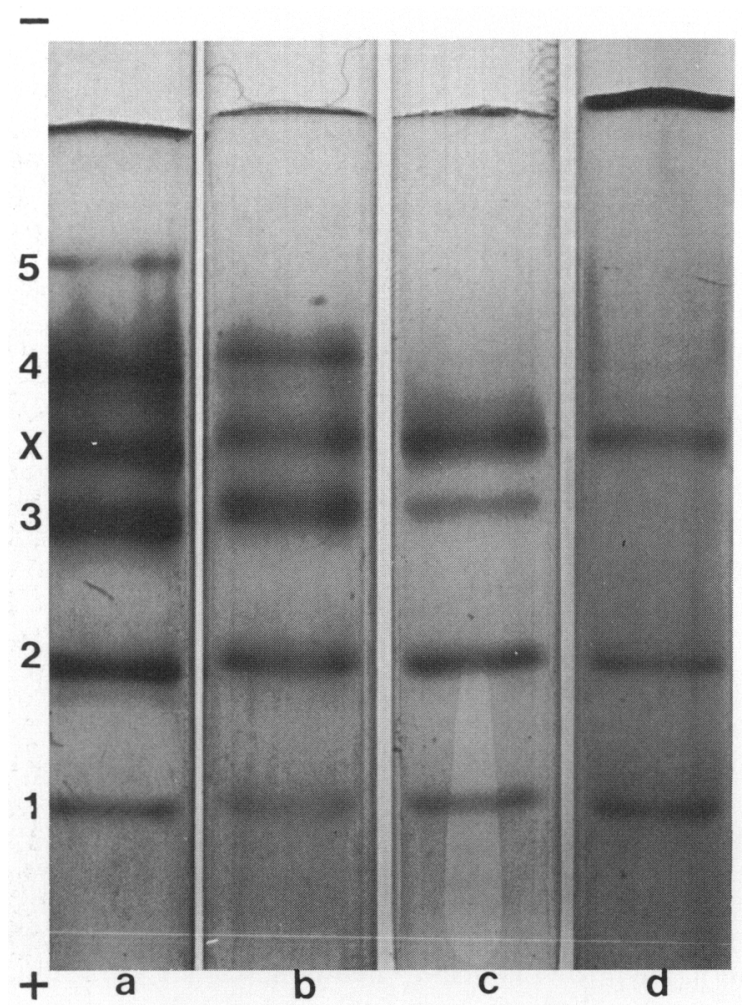

Fig. 3. Effect of heating at $65^{\circ} \mathrm{C}$ on the $\mathrm{LDH}$ isoenzymes in normal seminal plasma: (a) $0 \mathrm{~min}$, (b) $5 \mathrm{~min}$, (c) $15 \mathrm{~min}$, (d) $30 \mathrm{~min}$.

The same applies to C-subunits which behave like B-subunits when subjected to heat inactivation (Wilkinson \& Withycombe, 1965).

Seminal plasma samples incubated at $56-65$ and $70^{\circ} \mathrm{C}$ for $5-15$ and $30 \mathrm{~min}$, respectively, were examined to determine the temperature at which activity would disappear. In the samples incubated at $56^{\circ} \mathrm{C}$ for $5,15,30$ or $60 \mathrm{~min}$ before electrophoresis all bands were visible and no dramatic changes in $\mathrm{LDH}-\mathrm{C}_{4}$ activity could be measured with a specific substrate. The temperature of $70^{\circ} \mathrm{C}$ was too high even for the heat-stable $\mathrm{LDH}-1, \mathrm{LDH}-2$ and $\mathrm{LDH}-\mathrm{C}_{4}$ isoenzymes, and so no bands were visible.

In normal seminal plasma samples incubated at $65^{\circ} \mathrm{C}$, the LDH-5 band disappeared within $5 \mathrm{~min}$, the LDH-4 band and most of the LDH-3 fraction within $15 \mathrm{~min}$, while the intensity of the $\mathrm{LDH}-\mathrm{C}_{4}$ band remained unchanged. After $30 \mathrm{~min}$ only the $\mathrm{LDH}-1, \mathrm{LDH}-2$ and $\mathrm{LDH}-\mathrm{C}_{4}$ bands remained visible (Fig. 3). In the seminal plasma from infertile men with the additional bands incubated at $65^{\circ} \mathrm{C}$ for $15 \mathrm{~min}$ before electrophoresis, the LDH-5 and LDH-4 bands disappeared, but the extra bands and LDH-3, LDH-2 and LDH-1 were still visible (Fig. 4c). After heating at $65^{\circ} \mathrm{C}$ for $30 \mathrm{~min}$ the same results were obtained except for LDH-3, which was weakened but still present (Fig. 4d), although in the normal samples (Fig. 3d) the LDH-3 band had completely disappeared. This finding indicated that the LDH-3 region was actually occupied by an isoenzyme which was thermostable under the given conditions, i.e. by an isoenzyme containing B or C stable.subunits. These results seem to indicate that the LDH-3 isoenzyme is overlaid by some other thermostable isoenzyme. 


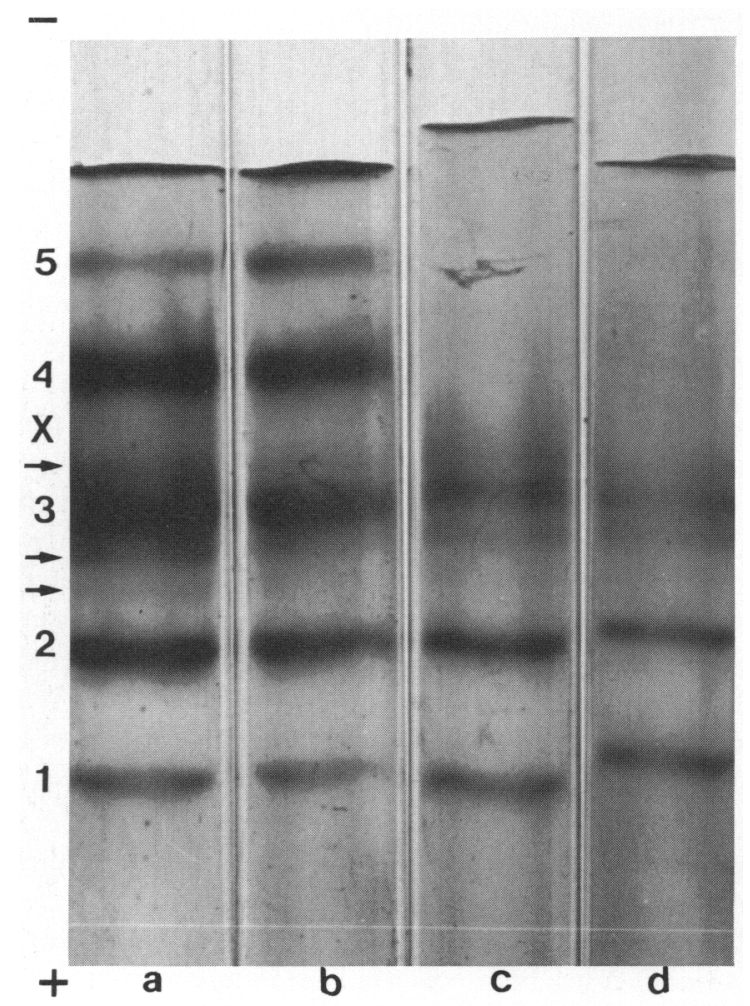

Fig. 4. Effect of heating at $65^{\circ} \mathrm{C}$ on the $\mathrm{LDH}$ isoenzymes in seminal plasma of infertile men: (a) $0 \mathrm{~min}$, (b) $5 \mathrm{~min}$, (c) $15 \mathrm{~min}$, (d) $30 \mathrm{~min}$.

\section{Discussion}

Although the existence of a single zone of human $\mathrm{LDH}-\mathrm{C}_{4}$ has been well documented in the literature, only three reports have been published on the occurrence of more than one tetramer containing C-subunits. In one of 41 human testes obtained by autopsy, Wheat \& Goldberg (1977) detected an unusual LDH isoenzyme pattern and provided evidence that the composition of this isoenzyme is consistent with an allelic variant of LDH-c. In 1984 Gavella et al. reported 3 additional LDH isoenzyme bands in the testes, spermatozoa and seminal plasma of some infertile men. Skude et al. (1984) also reported the discovery of additional $\mathrm{LDH}$ isoenzymes which, according to their location in the electropherogram, were believed to be tetramers in which the A and B subunits are combined with $\mathrm{C}$ subunits. These isoenzymes were found in the testes and spermatozoa of adult men, but could not be demonstrated in the seminal plasma. This finding is surprising, because, due to outward diffusion and spontaneous destruction of spermatozoa, $\mathrm{LDH}-\mathrm{C}_{4}$ can always be demonstrated in the seminal plasma if spermatozoa are present in the semen (Virji, 1985).

Since the unusual bands reported were detected at various sites in the electropherogram, suggesting different kinds of tetramers, the present study was conducted in an attempt to identify the composition of heteropolymers in our samples. Heat inactivation was used to detect differences in their physico-chemical features, while treatment with selective antisera allowed the identification of the type of heteropolymers involved.

The results obtained by immunoprecipitation have shown that antiserum to $\mathrm{LDH}-\mathrm{C}_{4}$ removes all the additional bands which do not react with antisera to LDH-A or LDH-B subunits. Therefore, 
our initial hypothesis that these additional isoenzymes were B-C heteropolymers was ruled out. Moreover, when the seminal plasma was treated with antiserum to A-subunits, even the LDH-3 $\left(A_{2} B_{2}\right)$ fraction, otherwise removable with this antiserum in normal seminal plasma, remained unprecipitated (Fig. 2c). Unlike other fractions containing A-subunits, the fraction in the $A_{2} B_{2}$ region also remained resistant to heat, which was additional evidence indicating the involvement of an isoenzyme with different characteristics. We can therefore conclude that some other isoenzyme is also present in the $A_{2} B_{2}$ region, i.e. in the electropherogram there are 4 instead of 3 new fractions, which, together, with a very weakly expressed band in the region normally corresponding to the LDH-C ${ }_{4}$ isoenzyme, give 5 fractions. The most cathodal of these 5 bands has the same electrophoretic mobility as $\mathrm{LDH}-\mathrm{C}_{4}$ in normal seminal plasma, while the band at the other extreme lies anodally to the $A_{2} B_{2}$ band, which represents an intermediate band. Since the activity is greatest in the intermediate band and decreases towards both extremes, showing a binomial pattern, an hypothesis can be put forward for the existence of two types of subunits most probably belonging to the C type, because they do not react with antisera to A-or B-subunits. These two types of subunits are arbitrarily named type I and type II subunits. Type I is most probably a polymer of four common $\mathrm{C}$ subunits, while type II comprises of four variant $\mathrm{C}^{*}$ subunits. Combination of the two types of subunits gives rise to binomially distributed 5 sperm-specific $\mathrm{LDH}$ isoenzymes: $\mathrm{C}_{4}$; $\mathrm{C}_{3} \mathrm{C}^{*} ; \mathrm{C}_{2} \mathrm{C}_{2}^{*} ; \mathrm{CC}_{3}^{*}$ and $\mathrm{C}_{4}^{*}$. Our results could be related to the findings of Wheat \& Goldberg (1977), who also detected an unusual pattern of 5 isoenzymes in a human testis, but in their electrophoretic pattern the variant tetramer was most cathodally situated, whereas the common $\mathrm{LDH}-\mathrm{C}_{4}$ was most anodally located.

Since our polyacrylamide gel electrophoresis was conducted under similar conditions, it appears that the same phenomenon was observed in both cases, although the characteristics of the isoenzymes were slightly different. Therefore, we accept Wheat \& Goldberg's (1977) explanation that an allelic variant of the LDH-C subunit must be involved. The pattern of 5 isoenzymes with binominally distributed activity is the one expected for a heterozygote at the LDH-c locus with both alleles co-dominantly expressed. The binomial distribution demonstrates that both alleles were functioning equally within the same cell. Both subunits must have been synthesized at the same time and at the same rate (Wheat \& Goldberg, 1977).

Regardless of the cause of the allelism at the clocus, the results of our study pose the question of functional activity of the C-C* isoenzymes formed and of the effect, if any, of their presence on sperm metabolism, mobility and fertilizing capacity. The fact that we found a decreased $\mathrm{LDH}-\mathrm{C}_{4} / 10^{8}$ spermatozoa ratio in the semen of infertile men of 980 compared to 1503 in 20 normal samples, and significantly reduced sperm motility and forward progression of spermatozoa (unpublished data) points to an impairment in the function of this specific lactate dehydrogenase isoenzyme, because disturbances in any of the compounds involved in the energetic machinery of spermatozoa will result in disruption of the overall process.

Therefore, further efforts should be directed to the study of the characteristics of variant isoenzymes and genetic studies that may contribute to the final resolution of the number of lactate dehydrogenase isoenzymes specific for the human spermatozoa.

We thank Professor E. Goldberg for anti-LDH-C 4 serum and M. Cvetkovic for help in preparing the rabbit anti-LDH-B ${ }_{4}$ serum.

\section{References}

Burgos, C., Coronel, C.E. \& Blanco, A. (1979) Substrate specificity of the lactate dehydrogenase isoenzyme $\mathrm{C}_{4}$ from human spermatozoa and a possible selective assay. J. Reprod. Fert. 55, 101-106.

Gavella, M., Cvitković, P. \& Skrabalo, Z. (1982) Seminal plasma isoenzyme LDH-X in infertile men. Andrologia 14, 104-109.

Gavella, M., Cvitković, P. \& Skrabalo, Z. (1984) Unusual forms of the human sperm lactate dehydrogenase isozyme. Cell. Mol. Biol. 30, 85-89. 
Goldberg, E. (1977) Isozymes in testes and spermatozoa. In Current Topics in Biological and Medical Research, pp. 79-124. Eds M. C. Rattazzi, J. G. Scandalios \& G. Whitt. Alan R. Liss, New York.

Markert, C.L. (1971) Isoenzymes and cellular differentiation. Adv. Biosci. 6, 511-526.

Skude, F.E., von Eyben, F.E. \& Kristiansen, P. (1984) Additional lactate dehydrogenase (LDH) isoenzymes in normal testis and spermatozoa of adult men. $\mathrm{Mol}$. Gen. Genet. 198, 172-174.

Usategui-Gomez, M., Wicks, R.W. \& Warshaw, M. (1979) Immunochemical determination of the heart isoenzyme of lactate dehydrogenase $\left(\mathrm{LDH}_{1}\right)$ in human serum. Clin. Chem. 25, 729-734.

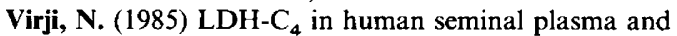
testicular function. I. Methodological aspects. Int. J. Androl. 8, 193-201.
Wheat, T.E. \& Goldberg, E. (1977) An allelic variant of the sperm-specific lactate dehydrogenase $\mathrm{C}_{4}(\mathrm{LDH}-\mathrm{X})$ isozyme in humans. J. exp. Zool. 202, 425-430.

Wheat, T.E. \& Goldberg, E. (1983) Sperm-specific lactate dehydrogenase $\mathrm{C}_{4}$ : antigenic structure and immunosuppression of fertility. In Isozymes: Current Topics in Biological and Medical Research, Vol. 113, pp. 113-130. Eds M. Rattazzi, J. G. Scandalios \& G. Whitt. Alan R. Liss, New York.

Wilkinson, J. H. (Ed.) (1970) Isoenzymes, p. 167. Chapman \& Hall, London.

Wilkinson, J.H. \& Withycombe, W.A. (1965) Organ specificity and lactate dehydrogenase activity. Biochem. J. 97, 663-668.

Received 29 April 1987 